\title{
MEDIACIÓN FAMILIAR CON PRESENCIA DE NIÑOS Y NIÑAS. UN ANÁLISIS DE MODELOS.
}

\section{Family Mediation with The Presence of Children. A Model Analysis}

Fecha recepción: 19 de enero de 2021 / fecha aceptación: 15 de mayo de 2021

Cómo citar este artículo:

Valdebenito Larenas C. (2021). Mediación familiar con presencia de niños y niñas. Un análisis de modelos. Revista Pensamiento y Acción Interdisciplinaria, 7(1), 146-165. http://doi.org/10.29035/pai.7.1.146

\section{Resumen}

Este documento ${ }^{2}$ aborda críticamente la intersección entre los modelos de la mediación aplicados a los temas de justicia de familia y las directrices de la doctrina de protección integral de la infancia para potenciar la figura del niño y niña como sujeto de derechos y su participación efectiva en los asuntos de su interés. Por ello asume la modalidad de ensayo teórico doctrinario que permite el levantamiento de un conjunto de interrogantes en torno a la temática.

Palabras claves: mediación familiar, niños y niñas, participación

\begin{abstract}
This document critically addresses the intersection between mediation models applied to family justice issues and the guidelines of the comprehensive child protection doctrine to enhance the figure of the child as a subject of rights and their effective participation in matters of interest to them. It therefore assumes the modality of doctrinal theoretical essay that allows the raising of a set of questions around the subject.
\end{abstract}

Keywords: family mediation, children, participation

\footnotetext{
1 Trabajadora Social. Magister en Gestión Colaborativa de Conflictos y Master en Estudios Avanzados de Mediación y Negociación. Doctoranda en Derecho. Académica de la Universidad Andrés Bello. Correo electrónico: caterine. valdebenito@unab.cl

2 Artículo en base a la ponencia presentada en el Congreso Federal, Internacional e Interdisciplinario "Los Derechos Humanos para la Niñez y la Adolescencia. Universidad de Buenos Aires. Mayo de 2020.
} 


\section{Introducción}

Desde las políticas públicas que orden los procesos de mediación en temas de familia, ha surgido desde finales del siglo XX, la inquietud acerca de la presencia de los niños y niñas en los procesos de la mediación familiar, en el entendido que, las temáticas que son abordadas en este dispositivo de atención legal, se refieren a las causas de pensión de alimentos, cuidados personales y relación directa, al tiempo que regula con ella progenitor/a que no vive con el niño/a.

Sin embargo, encontramos que existe una muy baja proporción de casos en que se inscribe de forma clara la participación de los hijos e hijas, tal es el caso que, en Argentina y Chile, no existen estadísticas que permitan dimensionar la incorporación de este segmento etáreo en los procesos que les involucran directamente. Lo anterior, nos lleva a preguntarnos ¿Cuál es la importancia que presenta la participación de los niños y niñas en los procesos de mediación en los temas de familia que les atañen directamente?

Por ello, parece adecuado, comenzar por revisar desde una mirada epistemológica y teórica, que se entiende por participación de los niños y niñas en los procesos de la mediación en temas de familia, para lo que revisaremos aquellos modelos teóricos que se han ocupado de forma explícita de esta temática, y que corresponden al Modelo de (Bolaños Cartujo, 2008, Cárdenas, 1999; Parkinson, 2005) los que son coherentes con las directrices de la Doctrina de Protección Integral a la Infancia y las normas legales vigentes en Chile en la misma materia (Vargas, 2002; Valdebenito, et al., 2018; Zato, 2015).

\section{Concepto de mediación familiar}

Para definir el alcance de la metodología de la mediación familiar, comenzaremos con la propuesta de la jurista Gladys Álvarez (2003) que señala que la mediación consiste en un procedimiento no adversarial en el cual un tercero neutral ayuda a las partes a negociar para llegar a un resultado mutuamente aceptable.

Por su parte, Moore (2006) se refiere a la mediación como la intervención en una disputa o negociación de un tercero aceptable, imparcial y neutral que carece de poder autorizado de decisión para ayudar a las partes en disputa a alcanzar su propio arreglo mutuamente aceptable.

Complementando estas definiciones, Sparvieri (1997) refiriéndose especialmente a la mediación familiar, enfatiza en las características no adversariales del procedimiento, que se centra en la búsqueda de los intereses de las personas involucradas y, que permiten orientar la búsqueda de la futura relación y el acuerdo, considerando a la base la permanencia de los vínculos de los distintos sistemas de la familia. Y que, de no existir estas bases, la mediación no es posible. 
El Consejo Consultivo de Francia, mediante el Libro Verde (CCE, 2002), ha convenido en definir a la mediación como un proceso de construcción y de reconstrucción del vínculo familiar sobre los ejes de la autonomía y de la responsabilidad de las partes afectadas por un conflicto, en cuyo proceso interviene un tercero imparcial, independiente, cualificado y sin ningún poder de decisión, para facilitar a través de entrevistas confidenciales, la reanudación de la comunicación entre las partes y la autogestión del conflicto dentro del ámbito privado familiar, teniendo en consideración la peculiaridad de las situaciones, su diversidad y la evolución de las relaciones familiares (Ortuño Muñoz, 2015).

Todas estas definiciones, coinciden en señalar ciertas premisas constitutivas del proceso de la mediación familiar, como la presencia de un/a tercero/a imparcial, llamado mediador/a que no tiene la capacidad de tomar decisiones, sino que su labor se orienta a la creación y facilitación de nuevas pautas comunicacionales entre las partes que viven un conflicto, con el fin que puedan buscar distintas soluciones a la disputa que presentan, en un ambiente resguardado y confidencial que genere espacios de dialogo que las personas no han podido desarrollar por sí solas.

\section{Orientación de la mediación familiar}

De acuerdo con lo señalado, parece estar claro que la incorporación de la mediación como mecanismo de gestión de los conflictos en los temas de justicia de familia, tiene una directa vinculación con el componente relacional y comunicacional que disponen los conflictos de los grupos sociales que mantienen relaciones permanentes y periódicas en el tiempo.

La existencia de las redes vinculares afectadas por los conflictos de las más diversas índoles que enfrentan las familias y los diferentes subsistemas que la componen, se ven afectadas en su calidad y cantidad por los juegos y disputas de poder, donde por ejemplo el conflicto de lealtades, la lucha para el ejercicio de ciertos roles, la disposición de los bienes económicos, el acceso a determinados integrantes del grupo familiar (Bolaños Cartujo, 2008), generando con ello, los escenarios conflictivos transaccionales que afectan la adecuada actuación de los/ las diferentes integrantes del grupo cuestionado.

En este sentido, la complejidad de la intervención que puede brindar la mediación familiar como dispositivo jurídico-psico-social, es más integrada a la realidad específica del grupo familiar considerando sus componentes personales, relacionales, sociales y del contexto cultural en que se desenvuelven.

Como ámbito de atención, construye "un espacio intermedio entre el mundo privado y el espacio judicial" (Bolaños Cartujo, 2008, p. 32), que permite que las personas involucradas en el conflicto puedan abordarlo de forma acompañada por el mediador/a manteniendo control sobre el poder de participación en la 
gestión de la situación, así como sobre la toma de decisiones que marcarán la ruta de continuidad o de término de la disputa.

Hay coincidencia entre los autores ya mencionados sobre aquello que determina que una familia pueda acceder a mediación va a estar dado por la manera en que se gestionó la separación o el divorcio, el estilo de la relación conyugal, la forma de comunicación del grupo familiar y la intensidad emocional asociada al conflicto. A ello, la experiencia de atención práctica de mediación (Valdebenito \& Donoso, 2018), nos permite señalar la necesidad de sumar los factores de tiempo transcurrido desde la separación física y las edades de los integrantes de la pareja conyugal.

Avanzando en ello, debemos agregar que el estudio de familias inmersas en procesos de separación o de divorcio, nos muestra que las posturas antagónicas que muestran los padres entre sí tienen efectos nocivos claros en la relación que establecen con los hijos e hijas, existiendo mayores alteraciones a la dinámica relacional previa (Valdebenito \& Donoso, 2018).

La separación del núcleo familiar siempre es dolorosa y plantea el fantasma de la pérdida de relaciones afectivas y de seguridad para sus integrantes, adultos, niños y niñas. Y éstos últimos, tienden a reaccionar con sentimientos ambivalentes hacia sus padres; por un lado, fuertes sentimientos de rabia e impotencia ante el adulto que abandona el hogar común y en paralelo el desarrollo de apegos intensos para el adulto que se queda (Bolaños Cartujo, 2008) buscando la protección y afecto que sienten perdidos.

Ante ello, la propuesta de la instalación de la mediación para la atención de los conflictos que acompañan la separación o divorcio de la pareja que tiene directa relación con los hijos e hijas, como son la tenencia o guarda, régimen comunicacional, pago de alimentos, manejo de pautas de crianza, ejercicio de roles parentales, parece adecuada en la perspectiva de su utilización dentro del sistema judicial de resolución de conflictos (Ortuño Muñoz, 2015).

Finalmente es adecuado señalar que la mediación no suple al sistema judicial, a los jueces o los diferentes operadores jurídicos que lo integran, siendo un mecanismo compatible que debe formar parte de la estructura de procedimientos judiciales destinados al abordaje y tratamiento humanizador de los conflictos que atraviesan las familias en proceso de separación y que afectan todos/as sus integrantes, y en especial a los niños y niñas. 


\section{Modelo de Mediación con familias que tienen hijos e hijas}

El conocido jurista argentino, Eduardo José Cárdenas, se desempeñó por largos años como juez en asuntos de familia, y desde su experiencia plantea la importancia de la mediación para la resolución de los conflictos existentes en la separación y/o divorcio de la pareja y el cuidado de los hijos e hijas del matrimonio, poniendo el énfasis en la relación de continuidad, aun cuando no vivan juntos.

\section{Bases del modelo de Cárdenas}

Cárdenas (1999), sustenta su modelo en la premisa del protagonismo de las partes o intervinientes. Siendo la mediación un escenario construido para promover cambios en la organización de la familia, que desequilibra el ejercicio del poder y corrige la forma de la toma de decisiones, enfocando la cualidad colaborativa para resolver el conflicto.

Como contraparte, la figura del profesional mediador/a, asume una figura de director/a de la reunión, que debe organizar el proceso de atención y al mismo tiempo procurar los espacios de participación para todos y todas los/las asistentes, sin suplir a alguno/a. Lo que implica que el/la profesional, pasa a ser un/a integrante más del circuito de atención, con un rol claro de facilitador, pero dentro del proceso de atención al mismo tiempo.

Un ejemplo de ello es que potencia que sean los y las asistentes los que definan consensuadamente los contenidos por los cuales se iniciaría el proceso de mediación, apuntando con ello a un término de proceso que sea duradero y confiable. Coloca un espacio de trabajo dispuesto en torno a los momentos de generación de confianza, apertura del conflicto, abordaje del problema, búsqueda de soluciones y el acuerdo.

\section{Niños y niñas dentro del proceso de mediación}

Eduardo Cárdenas explicita que en temas que se refieren a aspectos de manutención no es adecuado la presencia física de los hijos e hijas y que se encuentran suficiente y adecuadamente representados por los adultos responsables y que la manera de tenerlos presente se debe realizar de forma simbólica: por fotos y representaciones psicológicas a cargo de los padres. Poniendo hincapié en que son finalmente los adultos quienes tienen la tarea y responsabilidad de brindar alimentos a sus hijos e hijas, junto con evitar su parentalización.

El autor construye un apartado especial para evaluar la necesidad de la figura de los niños y niñas en los procesos de mediación (Cárdenas, 1999), poniendo el foco de su participación en dos temáticas específicas que les atañen directamente: la guarda y la relación con el padre/madre con quién no vive. 
Las razones de ello, son de tipo psicosocial enmarcadas dentro del principio de coparentalidad que propugna el derecho de familia y la doctrina de protección integral de la infancia, que muestran la importancia de comprender a la familia de padres separados, como una familia binuclear, es decir, una sistema extendido con dos casas; una casa de la madre y una casa del padre; con espacios subjetivos y legales abiertos que permitan la construcción de otras familias en su interior.

La manera de avanzar en este escenario desde las palabras de Cárdenas (1999), es la actuación desde la contracultura; esto es "abrir instancias de dialogo que permitan revisar los parámetros que se da por sentado en la cultura familiar clásica y que hace que se parta de supuestos que ponen roles estandarizados a hombres y mujeres" (p. 140), siendo un claro ejemplo, de cómo son las mujeres quienes se quedan naturalmente al cuidado de los hijos e hijas.

Desde este modelo, la revisión de dichos acuerdos sociales se postula como un necesario punto de partida para la instalación de la cultura de comunicación en todos los temas que tienen que ver con los hijos e hijas, de modo de hacer efectiva la coparentalidad. Asimismo, que se trabaje sobre el acuerdo que las decisiones son evaluables y cambiables conforme sea necesario para el grupo familiar binuclear, privilegiando la figura de la dupla paterna en la toma de decisiones.

La presencia de los niños y niñas dentro del proceso de mediación en temas de familia, para Cárdenas (1999) presenta aspectos positivos y negativos. Desde los aspectos de riesgo que es posible identificar, señala que puede involucrar a los hijos e hijas en el conflicto más de lo que ya están, puede oficializar que tomen partido por algún bando familiar, puede permitir que hablen por otra persona (padre o madre), puede que a un/a progenitor/a no le guste lo que diga y/o no se sienta querido/a por el hijo/a y que se retire de la audiencia frustrando la mediación, poniendo un cierto énfasis en los efectos que su presencia tendría en la participación de los padres.

Desde los aspectos positivos, comienza señalando que ello permite dar cumplimiento cabal a la Convención Internacional de los Derechos de los Niños, Niñas y Adolescentes (CIDN,1989) en lo relativo al derecho del/la niño/a de ser informado/a y ser oído/a en los asuntos que les atañen directamente, personaliza al//la hijo/a en la audiencia, permite que sea oído/a directamente por los padres de modo de presentar sus necesidades a los progenitores, permite a los padres conocer la imagen que el/la hijo/hija tiene de ellos, aporta en cerrar el dialogo del sistema familiar y puede añadir novedad de información al proceso de mediación. Encontrándose el foco del análisis centrado en los resultados de visibilizar a los hijos e hijas ante el sistema parental para apoyar la toma de decisiones.

En línea con lo anterior, de modo de potenciar los aportes y cuidar los riesgos identificados, propone una participación de los hijos e hijas de forma opcional y 
ordenada en el proceso de mediación familiar, siempre en los temas de guarda y relación directa y regular (Cárdenas, 1999).

Al referirse al criterio de Opcional, Cárdenas (1999) lo define como la decisión que tienen los padres de traer o no al/la hijo/a, a la audiencia de mediación. Coloca como criterio básico que son los dos padres quienes deben pensar que es adecuada y propicien la participación de los/las hijos/s, y que si uno/a de ellos/as no está de acuerdo no se produce la concurrencia.

Junto a ello, la claridad que la presencia de los/as hijos/as no está asociada al interés personal de uno de los padres, sino al convencimiento de los padres y del mediador/a que su participación es considerada útil para potenciar el desarrollo de la mediación familiar. Curiosamente, no aparece explícito en el planteamiento del autor la posibilidad que sean los/las hijos/as quienes pidan a los padres y/o al mediador/a participar en la mediación para poner en discusión temas de su interés.

El segundo criterio señalado por el jurista Cárdenas (1999), se refiere a la participación ordenada, como aquella que "habiendo sido acordada de realizar por los padres y el/la profesional, se construye un encuadre claro y especifico que organiza la presencia de los niños y niñas" (p.146-147).

Dicho encuadre considera que el mediador/a esta capacitado/a para entrevistar a los hijos/as; existe claridad del objetivo que los padres y/o el mediador/a buscan alcanzar con su participación y las características del dialogo a desarrollar, se ha definido el tipo de participación de los hijos/as conforme su nivel de desarrollo emocional y edad; la necesidad de sostener reuniones individuales o conjuntas si hay varios hijos/as de diferentes sexos y/o edades, la necesidad de que los padres estén o no presentes y el diseño de los roles de los adultos que estén presentes en la sesión, definir cuál es el lugar físico y tiempo para realizar la intervención y otros elementos que sean necesarios de considerar de acuerdo a cada caso en particular.

Dentro de este diseño de participación de los niños y niñas en el proceso de mediación, Cárdenas (1999), anticipa ciertos aspectos que creo necesario detallar para una mejor comprensión del enfoque que subyace a este modelo. Señala que la presencia de los niños y niñas debe estar alineada con el objetivo de poner a los padres en la situación de conducir acordadamente a la familia. Y por ello su participación será para recibir información acerca del proceso y al mismo tiempo recoger su opinión sobre tópicos específicos sin poder de decisión sobre ellos.

También debe estar presente en el relato del mediador/a el refuerzo a las figuras parentales, especialmente en la transmisión a los/las hijos/hijas, de que sus padres sienten micho afecto hacia ellos/as, que no tienen responsabilidad alguna en el proceso de separación en curso y con ello transmitir certezas que las 
decisiones que los padres puedan tomar se orientan a proporcionar las mejores condiciones para el grupo familiar que se encuentra en proceso de cambio.

También se espera que el/la profesional pueda apoyar a reestablecer el equilibrio de las relaciones de los/las hijos/as evitando y/o despejando comentarios de coaliciones de hijos/hijas con alguno de los padres, situando el foco en las necesidades e intereses propios de los niños y niñas como instancia de participación efectiva y no de representación de terceros/as.

Cárdenas (1999), propone que se cierre la presencia de los niños y niñas en la mediación, con la construcción de espacio ampliado de los padres e hijos/hijas donde el/la profesional y los padres puedan acoger a los menores, felicitarlos por su participación y poner en conocimiento de todos y todas, los puntos principales del dialogo realizado para evitar dobles discursos o fallidas interpretaciones, relevando con énfasis la mirada de futuro de la mediación.

Dentro de este modelo, parece surgir dos conceptos que no están explicitados y que son flexibilidad en el abordaje de cada situación, respetando el enfoque casuístico de la gestión colaborativa de conflictos, y también la noción de tiempo, entendida en los diferentes ritmos de conocimiento, necesidad de acoger los sentimientos y emociones producto de los cambios, la capacidad de elaborar propuestas y la diferencia en la toma de decisiones que pueden presentar las diferentes personas y en relación a los roles que desempeñan dentro del grupo familiar.

Finalmente, este modelo, abre la posibilidad de apoyar el trabajo del mediador/a, incluyendo un/a co-mediador/a, ojalá de diferente sexo y edad, que pueda aportar en el manejo de los niveles de conflicto en tratamiento, que no pierda de vista que el producto final, más allá de los acuerdos que puedan ser alcanzados, está en que los padres siempre deben ganar para realizar la conducción del grupo familiar binuclear. Donde la presencia de los niños y niñas presentaría la condición de opcional y ordenada, conforme los criterios que dispongan los padres, en primer lugar, y posteriormente el mediador/a, siendo la representación simbólica que posean los adultos participantes el criterio de definición al respecto, coherente con la tradicional mirada de protección que, presenta el derecho de familia mayoritariamente vigente. Lo anterior afecta de manera directa el cumplimiento del derecho de ser oído y de participación de los menores de edad, ya que limita su ejercicio en aras de la citada protección. 


\section{Modelo sistémico de L. Parkinson}

Dentro de la mediación familiar, ha sido Lisa Parkinson, quién acuñó el concepto de modelo ecosistémico de la mediación, considerando como foco el sujeto "familia". Señala en esta, que todos los integrantes de un grupo familiar son incluidos de manera directa o indirecta, puesto que todos son importantes en un sistema de decisión participativa, en que principalmente los padres y madres, están buscando obtener acuerdos en los temas de la crisis familiar y que generan consecuencias de diversa índole en la familia (Parkinson, 2005), particularmente en los hijos e hijas como un todo interrelacionado.

\section{Bases del modelo}

La característica principal de este modelo, se encuentra en la mirada compleja que desarrolla para iniciar, realizar y concluir la intervención con familias que tienen hijos e hijas y que están afrontando un quiebre del grupo familiar, que le permite realizar un mapeo de las distintas variables que están influyendo en la situación, para buscar vías sustentables de resolución.

Ello recoge lo que Saposnek (1983) investigó sobre las dinámicas interactivas complejas en que se encuentran las disputas familiares que afectan a los hijos e hijas, donde los comportamientos de éstos pueden apoyar y/o bloquear acuerdos de los padres, ya que los afectan de forma diferenciada.

Por ello, es que Parkinson, siguiendo a Berube y Lambert (2000), ha optado por el enfoque sistémico, que analiza a la familia como un todo, lo que aporta en la visibilización de todos sus integrantes, las relaciones entre éstos, los recursos disponibles, y en particular, las alianzas existentes.

Esto cobra especial relevancia, cuando se trata de una familia que está afrontando procesos de separación y/o divorcio, junto con ello, están los temas referidos al cuidado de los hijos e hijas, materia de suyo compleja en una familia estable, que debe lidiar con las tensiones del mundo laboral, social y familiar, y que recrudecen con especial fuerza en los procesos de ruptura (IFM, s.f.) 


\section{Niños y niñas en mediación familiar ecosistémica}

Es adecuado comenzar este punto, recordando que en las separaciones y/o divorcios, se produce el fenómeno que uno de los padres, mayoritariamente el hombre, se va del hogar común, lo que es vivenciado por los hijos e hijas como una pérdida que no siempre es explicada y/o comprendida en su totalidad. Aquello conlleva sentimientos de pérdida, estrés emocional y ansiedad que puede tomar diversas manifestaciones conforme los recursos y expresiones propias de cada niño o niña. Sin embargo, siguiendo a Parkinson (2005), pareciera ser que lo "que mayormente afecta a los hijos e hijas, es la manera en que los padres manejan la situación y la forma en que les explican los cambios que se están produciendo o se producirán" (p. 171).

Ello sucede, entre otras razones, por la estresante situación emocional en que se encuentran los adultos, la escasa información acerca de cómo se continuará la vida familiar y la relación con los hijos e hijas. Asimismo, dicha situación, dificulta las vías de comunicación en el subsistema conyugal, así como en el parento-filial. Pudiendo aparecer un conjunto de conductas desadaptativas en los niños y niñas, bajo la forma de regresiones, arrebatos de ira, descenso en el rendimiento escolar, por citar que corresponden a manifestaciones de pena y ansiedad experimentadas.

O, por el contrario, los niños y niñas, asumiendo la responsabilidad del quiebre, desarrollan conductas destinadas a resolver el conflicto entre los padres, conforme la información parcializada que disponen y/o los roles que le son asignados por sus padres.

Parkinson (2005) esquematiza en una tabla que presentamos a continuación, cuáles son las principales manifestaciones observadas en los niños y niñas, y propone tareas al mediador/a para su abordaje. 


\section{Tabla 1.}

\section{Papel del niño y niña}

\begin{tabular}{|c|c|}
\hline Papel del niño/a & Papel del mediador/a \\
\hline Mensajero/ intermediario/a & $\begin{array}{l}\text { Apoya a que los padres hablen directamente } \\
\text { entre sí, en vez de hacerlo mediante los hijos/as. }\end{array}$ \\
\hline $\begin{array}{l}\text { Reconciliador/a. Trata de volver a } \\
\text { unir a los padres. }\end{array}$ & $\begin{array}{l}\text { Facilita que los padres se pongan de acuerdo } \\
\text { sobre lo que es necesario explicar, les ayuda a } \\
\text { discutir sobre como tranquilizar a los hijos/as. }\end{array}$ \\
\hline $\begin{array}{l}\text { Pacificador/a. Le dice a cada padre y } \\
\text { madre, lo que cree quieren oír }\end{array}$ & Auxilia a los padres a resolver los conflictos. \\
\hline $\begin{array}{l}\text { Aliado reclutado por un padre/ } \\
\text { madre para obtener apoyo }\end{array}$ & $\begin{array}{l}\text { Ayuda a que los padres puedan llegar a acuerdos } \\
\text { y resuelvan sus conflictos para liberar a los hijos/ } \\
\text { as. }\end{array}$ \\
\hline Sujeto que toma las decisiones & $\begin{array}{l}\text { Ayuda a los padres a asumir la responsabilidad } \\
\text { respecto de las decisiones difíciles. }\end{array}$ \\
\hline $\begin{array}{l}\text { Víctima propiciatoria (teme ser } \\
\text { abandonado por ambos padres) }\end{array}$ & $\begin{array}{l}\text { Ayuda a los padres a darle certeza a los hijos/as } \\
\text { y a acordar los límites necesarios, definir reglas } \\
\text { etc. }\end{array}$ \\
\hline Confidente & $\begin{array}{l}\text { Auxilia a los padres en la reflexión sobre cómo } \\
\text { evitar cargar al niño/a. }\end{array}$ \\
\hline $\begin{array}{l}\text { Compañero/a sustituto/a } \\
\text { (reemplazando al cónyuge que se } \\
\text { marchó) }\end{array}$ & $\begin{array}{l}\text { Ayuda a los padres a sentirse más seguros para } \\
\text { que dependan menos del niño/a. }\end{array}$ \\
\hline $\begin{array}{l}\text { Cuidador/a sustituto/a (cuidando al } \\
\text { cónyuge y/o hermanos menores) }\end{array}$ & $\begin{array}{l}\text { Contribuye a reforzar la seguridad de los padres y } \\
\text { ayuda a entender las necesidades del niño/a. }\end{array}$ \\
\hline $\begin{array}{l}\text { Juez/a (incitado a culpar a un } \\
\text { progenitor/a) }\end{array}$ & $\begin{array}{l}\text { Discute con los padres como ayudar a que } \\
\text { los niños y niñas comprendan sin condenar a } \\
\text { ninguno de sus padres. }\end{array}$ \\
\hline $\begin{array}{l}\text { Fugitivo/a (escapa de casa, } \\
\text { conductas de riesgo) }\end{array}$ & $\begin{array}{l}\text { Habla con los padres sobre los riesgos y } \\
\text { preocupaciones. Busca una mayor implicación } \\
\text { parental. }\end{array}$ \\
\hline $\begin{array}{l}\text { En duelo por la familia pérdida } \\
\text { (Muestra el dolor que los padres } \\
\text { están reprimiendo) }\end{array}$ & $\begin{array}{l}\text { Ayuda a los padres a reconocer y a compartir la } \\
\text { tristeza además del enojo. }\end{array}$ \\
\hline
\end{tabular}

Fuente: Parkinson (2005).

Las situaciones descritas en la tabla anterior, nos muestra de forma concreta y reconocible, diversas manifestaciones que presentan los niños y niñas en los procesos de ruptura familiar, y al mismo tiempo, pautea un conjunto de tareas a 
ser ejecutadas por el mediador/a que se ocupa del caso, destinadas a reorientar el rol de los padres en la gestión de la crisis de separación.

Considerando el enfoque ecosistémico, los padres deben de tener presente que, así como ellos presentan tareas y quiebres en esta etapa, los hijos e hijas también los presentan, y que se pueden agrupar en: las ideas de la importancia de reconocer la ruptura de la relación de sus padres, para así desligarse del conflicto y de angustia parental y poder retomar sus propias actividades y rutinas (Wallerstein \& Corbin, 1989); asumir la pérdida y el duelo del cambio de estado de familia y aceptar la separación/divorcio, como estado permanente y avanzar en concebir esperanzas realistas sobre relaciones dignas de confianza.

En el mismo sentido, es tarea del profesional, presentar de forma explícita a los padres, las necesidades y requerimientos de los hijos e hijas de forma que puedan ser incorporadas de forma efectiva en el reencuadre global de la situación, siendo las más relevantes el poder proveer la información que les permita comprender lo que sucede, en un lenguaje y complejidad acorde a su edad y desarrollo evolutivo, de modo de confiar en la seguridad de la continuidad del amor y cuidado de sus padres y otras personas significativas de forma incondicional, así como la seguridad de su falta de responsabilidad en la ruptura de la pareja; otorgarles el permiso emocional de cada padre, para mantener la relación sana de afecto con ambos padres sin sentimientos de traición o culpa, junto con apoyar en mantener la rutina asociada a la escuela y en la casa entre otros.

El modelo ecosistémico, plantea que una buena forma de comenzar a abordar este punto, es considerando la mirada de parentalidad compartida o activa entre los padres que se están separando y/o divorciando, lo que puede incluir o no la consideración de la familia extendida en el marco de los recursos existentes.

Recordemos que la definición de parentalidad activa, está directamente ligada a la tuición o cuidado personal compartido entre ambos padres, de modo de asegurar la presencia colaborativa de estos en el proceso de desarrollo, crianza, educación y protección a los hijos e hijas comunes, independiente del domicilio en que habiten éstos, como ya se aclaró en el apartado anterior.

La mediación como proceso dialógico, permite a ambos padres, un espacio de encuentro y discusión sobre cómo abordar un plan de parentalidad compartida, de forma constructiva y con perspectiva de futuro. La práctica permite aseverar que los padres, normalmente están de acuerdo en la satisfacción de las necesidades de sus hijos e hijas y las diferencias se producen en la forma práctica de como satisfacerlas de forma adecuada.

Ahí es donde la figura del mediador/a debe estar atento/a, a apoyar la discusión práctica sobre los puntos que contempla el plan de parentalidad, pudiendo apoyar respecto del cuidado de la salud, controles médicos, dentales, atención en caso de enfermedad; el cuidado de la educación, elección de 
colegio, materiales escolares, apoyo en tareas, asistencia a reuniones de colegio, participación en eventos, la elección de la creencia religiosa, si corresponde, las vacaciones, celebraciones, regalos, excursiones, lo relacionado con el tiempo libre, así como los deportes y actividades de descanso; los contactos con familiares y amigos antiguos y nuevos de los padres.

Y en un plano más relacional, cuidar y acordar las formas de comunicación, en especial como transmitir la información respecto de acuerdos y modificaciones a los mismos; el tipo de disciplina, reglas y límites, respetando lo impuesto por el otro progenitor/a y acuerdos respecto de la responsabilidad eventual de otra persona, así como los diálogos sobre la seguridad y desarrollo del niño/a: educación sexual, drogas, entre otros (Parkinson, 2005).

Debemos tener claridad, que las intervenciones que son realizadas por el mediador/a, se efectúan utilizando la diversidad de técnicas y herramientas propias de la mediación familiar, de modo de apoyar el ejercicio reflexivo, destinado al desarrollo de un plan de parentalidad compartida adecuado para cada caso en particular.

Sin embargo, hasta ahora podemos observar, que el despliegue de trabajo dentro de la mediación está orientado al trabajo directo del profesional con los padres, en un escenario de atención y apoyo para y entre adultos, que se ocupan de los temas de interés de los niños y niñas, brindando con ello un espacio de presencia indirecta de los hijos/hijas en la mediación.

El espacio que contempla el modelo ecosistémico, para la participación directa de los niños y niñas en el proceso de mediación, se orienta a apoyar el ejercicio de la autoridad parental pudiendo tomar diferentes formas para ello.

Una de ellas, se refiere al apoyo para el traspaso de información de los padres hacia los hijos/hijas, respecto del conflicto en que se encuentran, las acciones realizadas y los acuerdos alcanzados para avanzar en su resolución. Donde la mediación, coloca un escenario imparcial y acogedor para ello, siendo la figura del/ la mediador/a un elemento de apoyo al ejercicio parental de preparar y entregar la información de forma amorosa, sin descalificarse o denigrarse entre ellos.

Hay una especial consideración sobre la preparación que requieren los padres para adelantarse y prever posibles reacciones de los hijos e hijas y cómo reaccionar frente a ellas, que implica un mejor manejo de las propias emociones ante las explosiones y manifestaciones de los hijos comunes. Y se operacionaliza, mediante una sesión conjunta de padres, mediador/a e hijos/hijas.

Un segundo escenario que contempla este modelo considera a la mediación, como un espacio de contención y de escucha de las necesidades y temores que viven los hijos/as en la crisis familiar. Privilegiando aquí el espacio, para entregar información de lo que suce de a los hijos/as, y al mismo tiempo de abrir una instancia 
donde éstos (los niños y niñas) puedan expresar sobre aquellos sentimientos, emociones, deseos, miedos e incertidumbres que están experimentando respecto de la situación familiar que están atravesando.

Este segundo dispositivo, es realizado mediante una reunión del mediador/a con cada hijo/a de forma privada, pone el énfasis en la necesidad de contención emocional de los niños y niñas, de modo de apoyar el ejercicio de la parentalidad compartida, incorporando a la vez la recogida de información proveniente del sistema filial que puede ser considerada o no por los padres al momento de la toma de decisiones, respecto de los acuerdos que guiarán el plan de parentalidad compartida.

El diseño presentado recoge de forma literal lo dispuesto en la Observación General No 12 del Instituto Interamericano del Niño y Niña (IIN, 2009), respecto del derecho que tiene el niño y niña a ser oído respecto de los asuntos de su interés, dando cumplimiento a la escucha activa para el acompañamiento emocional, sin que exista un espacio claro de la incorporación de sus opiniones y/o intereses en las resoluciones acordadas por los padres. Parkinson (2005), aporta que los estudios realizados en el Reino Unido, concluyeron que la mayoría de los niños y niñas opinaron que se les debe dar espacio para hablar de sus sentimientos y de ser escuchados, sin tener que decidir.

Los escenarios descritos, se muestran coherentes con la implementación de, al menos dos etapas de la escala destinada a describir los diversos niveles de participación de los afectados por decisiones de la autoridad del gobierno local (municipios), que Hart (1993) ha utilizado para medir la participación de los niños y adolescentes y que corresponden a la etapa de la consulta. Donde la autoridad (los padres) decide sola, pero antes consulta, la opinión del afectado o afectada (niño o niña) sobre las diversas alternativas posibles, sus ventajas e inconvenientes, al tiempo que, puede o no, mantenerle informado, sin entregar total o parcialmente parte de la decisión.

Mirado bajo este parámetro, se puede afirmar que la mediación ecosistémica no ha desarrollado de manera plena los elementos técnicos y procesales que permiten el cumplimiento de los derechos de los niños y niñas respecto a ser escuchados y que su opinión se considere en las decisiones sobre los asuntos de su interés y si se consideran los espacios adecuados para incorporar su participación efectiva, en el ejercicio y cumplimiento de las decisiones que les afectan de forma directa, encontrándose en un estado parcial de avance. 


\section{Modelo de mediación familiar transicional}

En España y producto de la sistematización del trabajo de mediación con parejas en proceso de separación y/o divorcio, se construye una propuesta de atención en mediación destinada a abordar las complejidades de la separación de la familia, en especial, referido a las temáticas de tenencia de los hijos e hijas y de la relación comunicacional con el padre/madre que no vive con ellos/ellas, que recibe el nombre de mediación transicional como ya lo anticipó el título de este apartado.

\section{Bases del modelo}

La propuesta de mediación transicional comienza con el reconocimiento que la separación de los padres corresponde a una crisis de desarrollo (Bolaños, 2008), que afronta el grupo familiar, la que dependiendo del manejo de los diversos conflictos que estén presenten, podrá resolverse en un escenario adaptivo o avanzará a convertirse en un espacio de hostilidad crónica que afecta negativamente el desarrollo de todos los integrantes de la familia en cuestión.

De acuerdo con Isaacs, et al. (1988), las crisis que viven las familias son parte constitutiva de su funcionamiento, y las pérdidas y sentimientos de dolor están presentes en todas ellas. Por lo que, la manera en que los adultos responsables puedan abordarlas y gestionarlas, para transformarlas en procesos de adaptación y de reencuadres de nuevas situaciones de vida, será vital en el desarrollo de todos.

La diferenciación de los sistemas conyugal y parental, es un adecuado punto de inicio para ello, de modo de separar los quiebres y dolores del primero en la diada conyugal, que pueda permitir la continuidad de los roles parentales que deben seguir estando presentes para los hijos e hijas.

Y desde este punto de partida, establecer que los procesos de mediación pueden ser desarrollados en un espacio prejudicial, judicial y postjudicial, que sabemos por lo general, permiten acceder a resultados más satisfactorios y sustentables para las familias afectadas basadas en decisiones acordadas de forma conjunta.

Ello en consideración, que los tiempos de los procesos legales son diferentes a los tiempos de los proceso psicosociales, siendo éstos últimos más extendidos en el tiempo (en su inicio y en su finalización), lo que afecta el comportamiento de la familia inmersa en un proceso judicial.

La mirada de Linares (1996) es recogida por este modelo de modo de tener presente, que si bien la conyugalidad y la parentalidad son atributos de la pareja, la manera en que cada integrante los percibe está mediatizada por los factores culturales personales y de su historia de vida, que se relacionan y van modificando en la interacción conyugal, y que se ven influidos por las variables sociales y 
ambientales, que configuran un sistema complejo en el ejercicio cotidiano de ambas condiciones, como se puede observar en el cuadro que sigue.

La mediación transicional, postula un abordaje de psico-jurídico, que permite una intervención más adaptada a la realidad conflictiva generada después del inicio del trámite contencioso, que ha instalado un lenguaje legal un tanto extraño y prácticas de confrontación y al mismo tiempo de pérdida de responsabilidad individual en el proceso en curso. De modo de apoyar el proceso de re-responsabilización de ambos padres, para que puedan alinearse con los hijos/as en el proceso de adaptación que todas afrontan para construcción de un nuevo tipo de familia y de relaciones de sus integrantes.

\section{Niños y niñas en la mediación}

La etapa de creación de nuevas opciones del modelo transicional contempla la alternativa de realizar como instancia opcional, la participación de niños y niñas, que ha denominado encuentro con el hijo o hija. Esta participación debe haber sido aceptada con ambos padres, lo mismo su acuerdo, sin que se detalle la forma y condiciones de tal acuerdo.

Dicha instancia, tiene por finalidad entregar información del proceso de trabajo realizado, de modo que el hijo/a pueda conocer de qué se trata la mediación, los esfuerzos realizados por ambos padres para asegurar la continuidad del ejercicio parental por separado; la construcción de un espacio de contención ante la vivencia de dolor presente en la ruptura, con el recuerdo de buenos momentos de relación parental y la seguridad que pueden ser retomados en el nuevo escenario parental y finalmente el promover el reencuentro de padres e hijos/as. En un lenguaje acorde a la edad y madurez de cada uno, considerando la necesidad de realizar entrevistas por separado en función de la edad, sexo y necesidades específicas de cada hijo o hija.

Y cierra el momento con un encuentro familiar, donde padres e hijos/as y el mediador/a negocian sobre los diferentes puntos en discusión, evitando los más conflictivos, de modo que los hijos/as puedan observar los esfuerzos realizados por los padres, más allá de las declaraciones escuchadas.

De forma previa a la presencia de los niños y niñas en la mediación, se entiende que las etapas de abordaje de conflicto han permitido que los padres puedan haber visibilizado de forma concreta y real el interés superior de sus hijos e hijas, generando la transición hacia el rol parental por sobre el conyugal.

Podemos concluir que este modelo de mediación también coloca un espacio muy acotado de participación de los niños y niñas en los procesos de mediación judicializados, siguiendo el mismo enfoque de protección existente en los modelos anteriormente presentados. 
Y que en aquellos casos que se genera la presencia de los niños y niñas, se deben ubicar en los niveles iniciales de la escala de participación de Hart (1993), que recoge Couso (2006), cubriendo los niveles de información y contención emocional.

\section{Algunas ideas al cierre}

Como se puede observar, los diferentes modelos presentados, colocan un gran énfasis en la protección del derecho a la intimidad y a la autonomía de la familia ante la intervención del Estado o los distintos dispositivos que lo integran, lo que se encuentra acorde a las directrices internacionales en la materia.

Asimismo, el lenguaje en que se construyen las representaciones de estos modelos teóricos recoge los conceptos base como protección a la niñez e interés superior del niño y niña, que también se encuentran en las normativas legales que regulan su aplicación y que se encuentran presentes en las bases presentadas.

Sin embargo, las diferencias comienzan a presentarse cuando surgen las miradas sobre cómo se hace realidad el escuchar a los niños y niñas en los procesos de mediación familiar, que se ocupan de los temas que los atañen directamente; apareciendo conceptualizaciones que recrean las nociones de parentalidad y poder parental por sobre los espacios de expresión de la niñez, basándose para ello en la discrecionalidad de los padres como los ejes de acción y de decisión.

Las nociones de opcional e imprescindible, pasan a ser complementadas y reemplazadas por los conceptos cuidado de la parentalidad, lo que mantiene en el centro la mirada a los padres: la forma que ellos definen el proceso, los recursos que disponen para abordar la temática y el espacio que entregan a los hijos e hijas, por citar algunos aspectos que se encuentran a la base de dichos planteamientos.

Vinculado al principio del derecho el niño y niña a ser oído y que sus opiniones sean consideradas de acuerdo a su edad y madurez, surgen las propuestas de trabajo de mediación con éstos, vinculadas a la entrega de información del proceso y/o de los acuerdos alcanzados, el foco de contención y apoyo emocional ante los quiebres familiares y la labor de apoyo al fortalecimiento de las figuras parentales en su ejercicio, respondiendo con ello a una lógica de acompañamiento a los padres más que a los hijos e hijas.

Y si profundizamos un poco más en dichos conceptos, podemos darnos cuenta de que existen ciertas aprehensiones de las competencias técnicas de los y las operadores, por un lado, pero por sobre todo, está la ausencia del abordaje de los principios rectores de la CDN en su interrelación y complejidad epistemológica y normativa en los procedimientos judiciales de la mediación familiar, siendo una tarea por abordar. Lo que no deja de llamar la atención, especialmente si 
consideramos otros espacios judiciales en el ámbito proteccional que, si los integran como son lo proteccional y lo penal juvenil.

Encontramos en ello que, la figura del niño y niña como sujeto de derechos sigue estando sujeta a una lógica de protección especial, que creemos muy necesaria por lo demás, que continua invisibilizando los procesos de avance que ha ido mostrando la doctrina de derechos de la infancia en la materia de participación para el ejercicio de sus derechos fundamentales, donde se han estado construyendo los espacios técnicos y normativos para favorecer el que sean escuchados y tomadas en cuenta sus opiniones e intereses así como apoyar la concreción de autonomía progresiva a través del ejercicio de toma de decisiones en ámbitos concretos de su mundo cotidiano, mirado ello como parte del ejercicio de ciudadanía del ser humano.

Cabe preguntarse entonces, ¿Cuáles son los pasos a nivel epistemológico, metodológico y normativo que debemos realizar para apoyar una interrelación propositiva del derecho de familia y el derecho de la niñez en los procesos de atención de mediación en temas de familia, que permita el reconocimiento efectivo de la participación de los niños y niñas para el ejercicio de sus derechos fundamentales? 


\section{Referencias bibliográficas}

Álvarez, G. (2003). La mediación y el acceso a la justicia. Editorial Rubinzal-Culzoni.

Berube, L., \& Lambert, D. (2000). La mediazone familiale. Etape par etape. CCHLTEE.

Bolaños Cartujo, I. (2008). Hijos alineados y padres alienados. Mediación familiar en rupturas conflictivas. Reus.

Cárdenas, J.P. (1999). La mediación en conflictos familiares. Luhmen.

Comisión de las Comunidades Europeas (2002). Libro Verde sobre las modalidades alternativas de solución de conflictos en el ámbito del derecho civil y mercantil. https://www.poderjudicial.es/cgpj/es/Temas/Mediacion/Normativa-yjurisprudencia/Normativa-europea/Libro-Verde-sobre-las-modalidadesalternativas-de-solucion-de-conflictos-en-el-ambito-del-derecho-civil-ymercantil

Convención Internacional de los Derechos de los Niños, Niñas y Adolescentes (1989). https://www.unicef.cl/archivos_documento/112/Convencion.pdf

Couso, J. (2006). El niño como sujeto de derechos y la nueva Justicia de Familia: Interés Superior del Niño, Autonomía progresiva y derecho a ser oído. Revista de Derechos del Niño, (3-4), 145-166. https://www.unicef.cl/archivos_ documento/192/revista\%20derechos\%203_4.pdf

Hart, R. (1993). La participación de los niños: de una participación simbólica a una participación auténtica. Ensayos Innocenti No4. UNICEF. https://www.unicefirc.org/publications/538-la-participaci\%C3\%B3n-de-los-ni\%C3\%B1os-de-laparticipaci\%C3\%B3n-simbolica-a-la-participaci\%C3\%B3n.html

Hinojal. S. (2005). Los menores ante la mediación. Cuadernos y Estudios de Derecho Judicial. CENDOJ.

IIN. (2009). Observación General №12 (2009): El derecho del niño a ser escuchado https://www.acnur.org/fileadmin/Documentos/BDL/2011/7532.pdf

International Family Mediation (s.f.) Código deontológico relativo a los procesos de mediación familiar internacional. Un proceso colaborativo. http://ifm-mfi.org/sites/default/files/CHARTER/SPANISH/Charter_IFM_ES.pdf

Isaacs, M., Montalvo, B., \& Abelsohn D. (1988) Divorcio difícil: Terapia para los hijos y la familia. Amorrortu.

Linares, A. (1996). Identidad y narrativa. Paidós. 
Moore, C. (2006). El proceso de mediación. Métodos prácticos para la resolución de conflictos. Gránica.

Ortuño Muñoz, P. (2015). La mediación como medio de solución de conflictos. En E. Pillado, \& F. Fariña, (Ed.), Mediación familiar. Una nueva visión de la gestión y resolución de conflictos vistos desde la justicia terapéutica (pp.1537). Tirant Lo Blanch.

Parkinson, L. (2005). Mediación familiar. Teoría y práctica: principios y estrategias operativas. Gedisa.

Saposnek, D. (1983). Mediating Child Custody Disputes: A Sistematic Guide for Familys Therapists, Cour Conselors, Attorneys and Judges. Jossey Buss Publishers.

Sparvieri, E. (1997). El divorcio. Conflicto y comunicación en el marco de la mediación. Biblos.

Valdebenito, C., \& Donoso, M. (2018). Modelos y herramientas de la mediación familiar. Editorial Académica Española.

Valdebenito, C, Rojo, A., \& Campillay, P. (2019). Mediación familiar y plan de parentalidad: mecanismos para el ejercicio del cuidado personal y corresponsabilidad en la paternidad y maternidadactiva. Revista de Mediación, 12(2), e10. https://revistademediacion.com/wp-content/uploads/2019/12/ Revista24-e3.pdf

Vargas, M. (2002). Los niños en la Mediación Familiar. Revista de Derechos del Niño, 8(1), 137-166. https://doctrina.vlex.cl/vid/ninos-mediacionfamiliar-651168985

Wallerstein, J., \&. Corbin, S. (1989). Daughters Of Divorce: Report From a TenYear Follow-Up. American Journal of Orthopsychiatry, 59(4), 593-604. https://doi.org/10.1111/j.1939-0025.1989.tb02749.x

Zato Echeverria, M. (2015). Una aproximación al mapa de la mediación en la Unión Europea. Revista de Mediación, 8(1), 72-83. https://revistademediacion.com/ wp-content/uploads/2015/07/Revista-Mediacion-15-8.pdf 